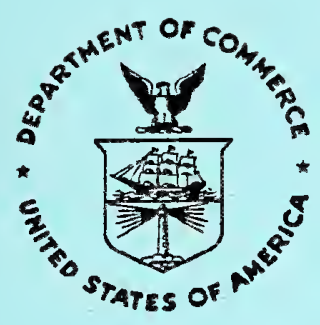

\title{
An Approach to Accurate X-Ray Mask Measurements in A Scanning Electron Microscope
}

Michael T. Postek, Robert D. Larrabee and William J. Keery

U.S. DEPARTMENT OF COMMERCE

National Institute of Standards and Technology

(Formerly National Bureau of Standards)

Microelectronics Dimensional Metrology Group

Precision Engineering Division

Gaithersburg, MD 20899

January 1989

Prepared for

The Naval Research Laboratories

Washington, DC 



\section{NISTIR 89-4047}

\section{An Approach to Accurate $X$-Ray Mask Measurements in A Scanning Electron Microscope}

Michael T. Postek, Robert D. Larrabee and William J. Keery

U.S. DEPARTMENT OF COMMERCE

National Institute of Standards and Technology

(Formerly National Bureau of Standards)

Microelectronics Dimensional Metrology Group

Precision Engineering Division

Gaithersburg, MD 20899

January 1989

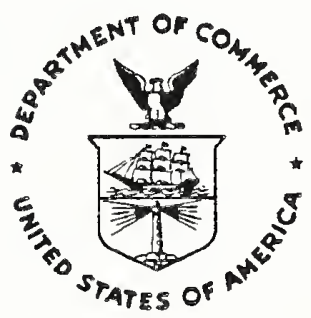

National Bureau of Standards became the National Institute of Standards and Technology on August 23, 1988, when the Omnibus Trade and Competitiveness Act was signed. NIST retains all NBS functions. Its new programs will encourage improved use of technology by U.S. industry.

Prepared for

The Naval Research Laboratories

Washington, DC

\section{U.S. DEPARTMENT OF COMMERCE}

Robert A. Mosbacher, Secretary

Ernest Ambler, Acting Under Secretary for Technology

NATIONAL INSTITUTE OF STANDARDS

AND TECHNOLOGY

Raymond G. Kammer, Acting Director 



\section{Table of Contents}

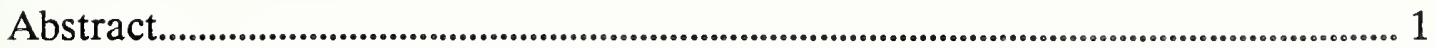

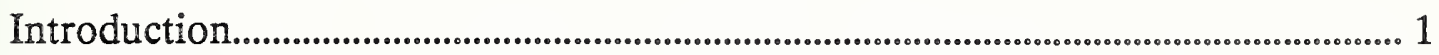

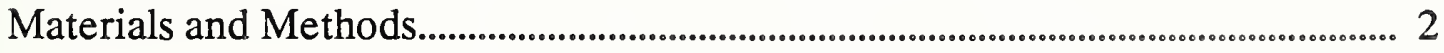

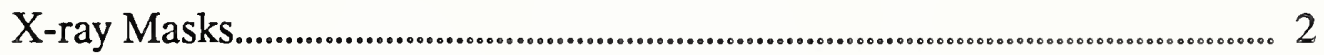

Transmitted Scanning Electron Microscope Mode................................. 3

Modeling.................................................................................................................... 3

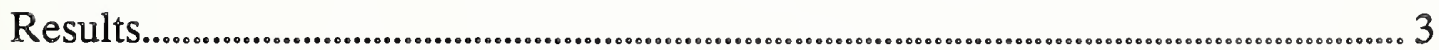

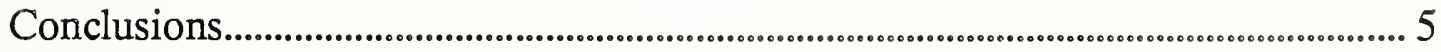

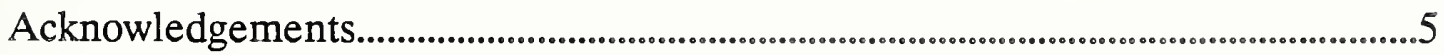

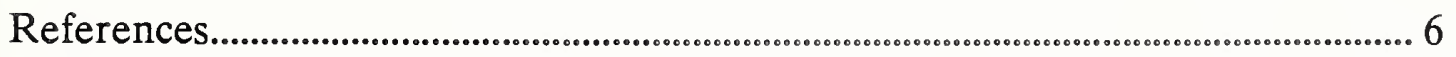

\section{List of Figures}

Figure 1. Schematic drawing of an X-ray mask

Figure 2. Measurements of a 0.5 micrometer line................................................. 8 



\section{Preface}

This work was conducted as part of the Microelectronics Dimensional Metrology program at the National Institute for Standards and Technology (NIST). The portion of the work described in this report was partially supported by the Naval Research Laboratories, Washington D. C. under Contract \# N0017388WR80360. The contract was monitored by Dr. Marty Peckerer of the Naval Research Laboratories and the point of contact at NIST for information about the technical elements of this project was Dr. Michael T. Postek of the Microelectronics Dimensional Metrology Group at the National Institute of Standards and Technology, Gaithersburg, Maryland. 



\title{
AN APPROACH TO ACCURATE X-RAY MASK MEASUREMENTS
}

\author{
IN A \\ SCANNING ELECTRON MICROSCOPE \\ Michael T. Postek, Robert D. Larrabee and William J. Keery \\ Microelectronics Dimensional Metrology Group \\ Precision Engineering Division \\ National Institute of Standards and Technology \\ Technology Building A-347 \\ Gaithersburg, MD 20899
}

\begin{abstract}
This paper presents the concept and some preliminary experimental data on a new method for measuring critical dimensions on masks used for $\mathrm{x}$-ray lithography. The method uses a scanning electron microscope (SEM) in a transmitted-scanning electron microscope (TSEM) imaging mode and can achieve nanometer precision. Use of this technique in conjunction with measurement algorithms derived from electron beam interaction modeling may ultimately enable measurements of these masks to be made to nanometer accuracy.
\end{abstract}

\section{INTRODUCTION}

The design and proper performance of state-of-the-art semiconductor devices ultimately resides in the strict adherence to design rules. With design rules in the submicrometer region, and with the necessity to adhere to specified critical dimensions, the measurement of these dimensions becomes extremely important. Presently, there are no satisfactory methods for providing traceability to the National Institute of Standards and Technology (NIST) for critical-dimension measurements on the state-of-the-art X-ray masks under development for a wide variety of military and commercial applications. This situation has developed because the evolution of submicrometer dimensions by the semiconductor industry has occurred faster than the parallel development of the dimensional metrology and the standards required to support this evolution. The areas of greatest need are those areas where the fabrication art has equalled or surpassed the required metrological art. 
NIST has maintained an active microelectronics dimensional metrology program since the late 1970's. Its optical dark-chrome photomask standards (SRM 474 and 475) cover the linewidth range from 0.9 to 10 micrometers and a future standard SRM 473 will extend this range from 0.5 micrometer to 30 micrometers. Unfortunately, the corresponding standards for features on photoresist, semiconductor devices and X-ray masks present totally different, and much more difficult, metrological problems in both the optical ${ }^{1}$ and the scanning electron microscopes. ${ }^{2}$ These problems arise because the features are not thin compared to the wavelength of illuminating light (in the optical microscope) or the electron scattering range (in the scanning electron microscope), and this results in uncertainties in the location of the edges in the optical or SEM image of the feature being measured. Because of these problems, the present photomask standards are totally inadequate for use in calibrating systems used in the measurement of dimensions of nonphotomask features (e.g., features on semiconductor devices or on X-ray masks). However, NIST does have active programs to develop the necessary metrological tools and techniques for nonphotomask features in both the optical and scanning electron microscopes. The X-ray mask work presented here is one method under development to measure, and ultimately certify, X-ray mask standards in the metrological scanning electron microscope under development at the Institute。 ${ }^{3}$ This method has the potential advantage of avoiding, or at least minimizing, the basic limitations imposed by the electron beam interaction effects normally encountered in conventional methods of dimensional metrology in the SEM.

By their nature, masks for X-ray lithography present a measurement subject unique from most (if not all) other samples used in semiconductor processing because they are, by design, essentially either electron opaque or essentially electron transparent depending upon the area being viewed. This characteristic can be utilized to make precise and accurate measurements since the sample can be viewed in the transmitted scanning electron microscope (TSEM) mode. ${ }^{4}$ In the TSEM mode, electron beam/specimen interaction modeling becomes far less difficult than in the modeling of typical secondary electron images of opaque objects since, to a first approximation, the TSEM mode is concerned only with unscattered high-energy electrons. The scattered high-energy beam electrons and the low-energy secondary electrons can be excluded from the detector and, therefore, need not be modeled.

\section{Materials and Methods}

X-Ray Masks. Masks for X-ray lithography are made in several ways depending upon the manufacturing process; however, except for the thickness of the supporting thin-layer (essentially electron transparent) substrate, this should have little bearing on the present technique. The masks used in this study were composed of a substrate of approximately 1.0 micrometer of silicon upon which the structure was patterned in a layer of gold approximately 0.4 micrometer thick (Figure 1). The gold patterned features of the mask have some edge irregularities as observed in an SEM but, for the most part the edges are reasonably vertical in cross section. Given these materials and dimensions, the electron 
range at $30 \mathrm{keV}$ accelerating voltage is approximately 10 micrometers in the substrate and approximately 2 micrometers in the patterned layer. This means that for every 10,000 electrons impinging on the mask, approximately 9550 electrons are transmitted through the silicon substrate while only about 35 electrons are transmitted through the gold/silicon structure, and of these 35 electrons, the majority will have been highly scattered and will, thus leave the sample at a large angle relative to the incident beam. Therefore, the region masked by the gold is almost opaque to the beam electrons of this energy and the silicon substrate sufficiently transparent so that the electron beam travels through it with limited attenuation and deviation.

Transmission Scanning Electron Microscope Mode. In the conventional secondary-electron imaging mode of the SEM, as demonstrated by Postek et al. $^{5}$, the edge of features have a characteristic edge broadening (and thus location uncertainty) due to the electron-beam interaction; whereas in the TSEM mode of operation, the transmitted electron image is virtually unbroadened by this interaction and without the large uncertainty in edge location. The TSEM mode of operation is readily accomplished in the SEM by the installation of a detector below the sample. The electron beam travels through the electron transparent portions of the sample and impinges directly on the detector. The type of electron detector used is not critical to the outcome and can be either a scintillator, solid state diode, channelplate or other suitable unit.

Electron Beam Modeling. The region near the feature edges, where any electron scattering effects are the greatest, has been modeled using an electron-beam scattering model developed by Newbury and Myklebust (personal communication). That model is presently undergoing further refinements such as proximity effects of neighboring lines and other geometric effects to improve its applicability for the present purpose. These refinements are needed because, of the initial 10,000 electrons striking the gold/silicon structure, $45 \%$ become backscattered electrons and any subsequent effects of these electrons on the measured image must be considered.

\section{Results}

The ideal transmitted electron signal in the TSEM Mode is formed from the undeviated and minimally scattered component of the electron beam. The form of this signal is determined, in part, by the solid angle of collection by the detector, which can be made quite small. Therefore, the electron-beam modeling is simplified relative to other types of opaque semiconductor samples measured in secondary electron imaging mode. ${ }^{2}$ The simplification arises because only the transmitted electrons need to be modeled, and even those electrons are restricted by the geometry and placement of the electron detection system. Therefore, the modeling required to describe the results and interpret the SEM image need not be as exact as in the case of secondary electron imaging. Those electrons undergoing large-angle inelastic interactions with the sample can be eliminated through aperturing of the 
transmitted-electron detection system, and backscattered electron contributions can be minimized by improved specimen chamber/sample internal geometry. Further, the solid-state diode detector used for this study has a threshold excitation energy about 3-5 $\mathrm{keV}$ so those electrons having undergone high energy loss due to inelastic collisions, but with a trajectory leading to the detector, are also filtered from the measured signal. Secondary electrons generated at the backside of the substrate by the high-energy transmitted electrons are likewise filtered and go undetected. Since the number of electrons reaching the transmitted electron detector is rather small relative to a secondary-electron image, the gain and performance of the detector/amplification system must be high. Current solid-state diode systems require improvement in this area in order to achieve high gain and wide bandwidth with good signal-to-noise ratio for the small beam currents at small incident spot diameters. Work presented here comparing measurements between the standard secondary electron detector and the transmitted electron detector was optimized for an existing less-efficient diode detector. This required a larger than desired electron beam spot size and thus provided an undesirably large edge uncertainty, but sufficed to demonstrate the present technique.

Measurements of a given area of an X-ray mask taken at 50,500x (where the pixel resolution is approximately 0.004 micrometer) demonstrate that although the measured pitch of the lines remains relatively constant (Figures $2 a$ and $b$ ), the width of the lines varies between secondary electron imaging and transmitted electron imaging (Figures $2 \mathrm{c}$ and $\mathrm{d}$ ). The wider measurement result for the transmitted image (Figure 2d) is likely due to the width measurement corresponding to an undetermined point near the actual base of the line (the area most important to the lithography), whereas the secondary electron measurement corresponds to an unknown threshold point which may be near the top of the line or some arbitrary location on the line edge. The actual position is unknown due to the fact that adequate electron beam interaction modeling is not available to identify the true edge location for secondary electron imaging. Furthermore, due to the capricious nature of secondary electron emission and beam-specimen interactions, it is not clearly known where the actual measured secondary electrons originated. ${ }^{2}$

In order to assess the magnitude of the edge broadening induced on the measurement due to the large beam diameter required to overcome signal-to-noise limitations, the electron beam diameter was measured using NIST (NBS) SRM 2069 SEM Performance Standard mounted at the same location as the X-ray mask sample and measured under the same SEM operating conditions. Comparison of the slope of the line in both the secondary electron and transmitted electron modes to the profiles obtained from similar conditions using SRM 2069 demonstrates that the slope uncertainties are directly related to the beam diameter. ${ }^{6}$ Adjustment of the condenser lens of the SEM increased the measured edge slope thus resulting in a decrease in spot diameter as measured using NBS SRM 2069 in the secondary electron collection mode. However, noise limitations prevented similar work with the diode detector. ${ }^{6}$ The dependence of edge slope on spot diameter has been modeled by Matsukawa and Shimizu ${ }^{7}$, and the results of this work are consistent with their results. 
Another approach to the detection of the transmitted electrons would be to incorporate a dark-field electron-detection system (Joy, personal communication) in those cases where the detector is susceptible to unattenuated electron beam damage (i.e., burning of a scintillator phosphor). The use of either a scintillator detection system or a channel electron multiplier detection system would probably result in improved signal-in-noise for any given beam current (i.e. spot size).

\section{Conclusion}

Measurement of X-ray masks in the transmitted-electron imaging mode presents a unique opportunity to obtain more precise and, ultimately, more accurate measurement capability once algorithms based on the electron beam modeling data have been developed. The results of this work, to date, indicate that the present technique could result in an edge location uncertainty as low as 0.05 micrometer without modeling and as low as 0.01 micrometer with modeling. This performance could be improved by refinements in the detection and amplification of the TSEM signal, an improvement in the number of pixel points available, and in the use of a high-brightness high-resolution field-emission electron source. Incorporation of this technique to develop and certify $\mathrm{x}$-ray mask standards would require significant modification of the NIST metrology instrument and laser interferometer stage $^{3}$ designed for traceability to the national standards of length. Methods of funding such a program are presently being sought.

Unfortunately, metrology based on the transmitted-electron image is not readily adaptable to opaque specimens such as photoresist on silicon wafers where the need for standards and precise measurements is presently the greatest. However, a modification of the present technique could be used to make measurements on such samples if standard surface replication techniques for these types of samples are developed and the transmission measurements performed on the replica. These two methods (i.e. transmission measurements on actual specimens or replicas) are being explored in the NIST Microelectronics Dimensional Metrology Program to determine if they can serve as a method of calibrating submicrometer dimensional standards.

\section{Acknowledgements}

The authors would like to acknowledge and thank Hampshire Instruments, Inc. Marlborough, MA for the use of the X-Ray Lithography mask used in this study; Drs. Dale Newbury and Robert Myklebust for their assistance in the electron beam modeling program; Dr. David Joy for his recommendations for the use of an annular detector for collection of the transmitted electrons. 


\section{References}

1) Nyyssonen, D., and Larrabee, R.D., "Submicrometer Linewidth Metrology in the Optical Microscope." J. Res. Natl. Bur. Stand. 92(3): 187-204. 1987.

2) Postek, M.T., and Joy, D.C., "Submicrometer Microelectronics Dimensional Metrology: Scanning Electron Microscopy." J. Res. Natl. Bur. Stand. 92(3): 205-228. 1987.

3) Postek, M.T., Larrabee, R.D., and Keery, W.J., "Scanning Electron Microscope Linewidth Measurement Standards Program at the National Bureau of Standards. EMSA Bulletin 17(2): 59-64. 1987.

4) Postek, M.T., Howard, K.S., Johnson, A. and McMichael, K., "Scanning Electron Microscopy - A Student's Handbook." Ladd Research Industries, Burlington, Vermont, pp. 305. 1980.

5) Postek, M.T., Keery, W.J., and Larrabee, R.D., "The Relationship Between Accelerating Voltage and Electron Detection Modes to Linewidth Measurements in an SEM. Scanning 10:10-18. 1987.

6) Postek, M.T., Larrabee, R.D., and Keery, W.J.,"A new approach to accurate X-ray mask measurements in a scanning electron microscope." (in review).

7) Matsukawa, T. and Shimizu, R., " A New Type Edge Effect in High Resolution Scanning Electron Microscopy." Jap. J. Appl. Phys. 13(4):583-586. 1974. 


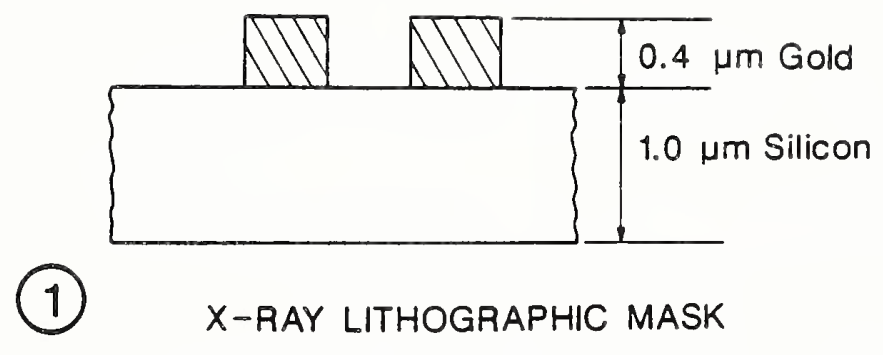

Figure 1. Schematic drawing of the $x$-ray mask used in this study. 
NBS-114A (REV. 2-8C)

U.S. DEPT. OF COMM.

BIBLIOGRAPHIC DATA

SHEET (See instructions)

1. PUBLICATION OR REPORT NO.

NISTIR $89-4047$
2. Performing Organ. Report Nof 3. Publication Date

MARCH 1989

4. TITLE AND SUBTITLE

An Approach toAccurate X-ray Mask Measurement in a Scanning Electron Microscope

5. AUTHOR(S)

Michael T. Postek, Robert D. Larrabee, and William J. Keery

6. PERFORMING ORGANIZATION (If joine or other than NBS, see inseructions)

7. Contract/Grant No.

NATIONAL BUREAU OF STANDARDS

U.S. DEPARTMENT OF COMMERCE

GAITHERSBURG, MD 20899

9. SPONSORING ORGANIZATION NAME AND COMPLETE ADDRESS (Street City State $_{*}$ ZIP)

10. SUPPLEMENTARY NOTES

Doeument describes a computer program: SF-185. FIPS Software Summary, is atcached.

11. ABSTRACT (A 200-word or less foctual summary of most significant information. If document includes a significant bibliogrophy or literature survey. mention it here)

This paper presents the concept andsome preliminary experimental data on a new method for measuring critical dimensions on masks used for $x$-ray lithography. The method uses a scanning electron microscope (SEM) in a transmitted-scanning electron microscope (TSEM) imaging mode and can achieve nanometer precision. Use of this technique in conjunction with measurement algorithms derived from electron beam interaction modeling may ultimately enable measurements of these masks to be made to nanometer accuracy.

12. KEY WORDS (Six 20 iwelve entries; olphabetical order; copicalize only proper names; and separate key words by semicolons) CD; critical dimension; dimensinal metrology; integrated circuit; 1ithography scanning electron micrscope; SEM; $\mathrm{x}$-ray mask

13. AVAILABILITY

[ Unlimited

For Official Distribution. Do Not Release to NTIS

$\exists$ Order From Superintendent of Documents, U.S. Government Printing Office, Washington, D.C. 20402.

[X] Order From National Technical Information Service (NTIS). Springfield, VA. 2216I
14. NO, OF

PRINTED PAGES

13

15. Price 
